\title{
Electromagnetic anapoles of a Cartesian expansion of localized electric currents
}

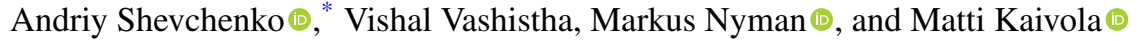 \\ Department of Applied Physics, Aalto University, P.O. Box 13500, FI-00076 Aalto, Finland
}

(Received 22 September 2020; revised 17 November 2020; accepted 7 December 2020; published 18 December 2020)

\begin{abstract}
Electromagnetic anapoles are nonradiating oscillations of electric charge. They are known to be formed by a combination of an ordinary and a toroidal multipole. In this work, we introduce a different family of anapoles based on a Cartesian electric-current multipole expansion and propose a way to create the lowest-order, centrosymmetric member of this family. We also find a nontoroidal multipole structure that radiates as an electric dipole despite its zero total dipole moment. Radiation-free charge oscillations are interesting not only in view of their unique fundamental properties, but also in view of practical applications, e.g., in near-field-based optical sensing and detection.
\end{abstract}

DOI: 10.1103/PhysRevResearch.2.042043

The electromagnetic multipole expansion is a useful tool for characterizing electromagnetic fields radiated or scattered by small particles, because any such field can be unambiguously expanded into orthogonal components produced by equivalent elementary point multipoles located at the center of the particle. The real electromagnetic excitations in the particle can differ substantially from the charge oscillations of the obtained multipoles. In most cases, the real excitations are more complex. As an example, the field radiated by a toroidal multipole excitation-omitted in the classical multipole expansion-is the same as that of the corresponding ordinary multipole [1-3]. Therefore, in the expansion of the radiated field, a toroidal dipole will show up simply as an ordinary dipole, even though the total dipole moment of this excitation (integrated over the volume of the particle) is equal to zero. If one then superimposes a toroidal dipole with an ordinary dipole that radiates the same, but the out-of-phase field in the absence of other multipoles, the overall radiation disappears, manifesting a completely dark anapole excitation. The classical multipole expansion of the field in this case yields zero expansion coefficients for all multipoles. Anapole excitations based on toroidal multipoles have recently been intensively studied, and their surprising properties and potential applications have been widely discussed [4-15]. One of the most intriguing features of anapoles is their ability to produce zero-energy vector-potential waves [7-9], with possible implications connected to the Aharonov-Bohm effect. Electromagnetic anapoles also show a strong near-field enhancement in the absence of far-field radiation that can be used, e.g., for near-field imaging and detection with extraordinary sensitivity [11-15].

\footnotetext{
*Corresponding author: andriy.shevchenko@aalto.fi

Published by the American Physical Society under the terms of the Creative Commons Attribution 4.0 International license. Further distribution of this work must maintain attribution to the author(s) and the published article's title, journal citation, and DOI.
}

Along with the multipole expansion of the radiated field, one can expand the electric current density in the particle into the electric-current multipoles [16-18]. In this work, we use this type of multipole expansion and construct pure and hybrid electromagnetic anapoles based on it. Pure anapoles consist of electric-current multipoles of the same order, while hybrid anapoles are combinations of current multipoles of different orders. The lowest-order anapole (that can be considered as a structural unit of higher-order pure anapoles) is highly symmetric compared to the anapole that includes a toroidal dipole. This fundamental anapole represents a pulsating oscillation of a spherically symmetric charge distribution. If, for example, the excited particle is an atom with a spherical electron cloud (the $s$ orbital), the excitation would appear as a periodic change of the electron-shell radius without breaking the spherical symmetry. Because this oscillation does not radiate (see, e.g., [19]), it should not be possible to excite it externally. However, we show that an equivalent charge oscillation can be excited in a noncentrosymmetric particle, such as a six-metalnanorod scatterer that we have designed for this purpose. As a figure of merit for such anapole designs we introduce a ratio of the total electromagnetic power radiated by an electric dipole to that of the designed anapole with the same integrated amplitude of the electric current density in the particle. For our design, the figure of merit (FOM) can reach the level of $10^{3}$.

In the following, we first briefly introduce the electriccurrent multipole expansion. Then we describe the construction of electromagnetic anapoles and present the design of a plasmonic particle in which the fundamental anapole can be excited. The summary of the work is presented at the end of the Rapid Communication.

In the electric-current multipole expansion, the electriccurrent density in a radiating or scattering particle is expanded into orthogonal Cartesian multipoles composed of elementary point currents [16]. These currents can be generated by applying multiple spatial derivatives to the Dirac delta function $\delta(\mathbf{r})$. The first-order point-current density distribution (corresponding to a classical electric dipole moment $\mathbf{D}$ ) is represented by the amplitude $\mathbf{J}^{(1)}(\mathbf{r})=-i \omega \mathbf{D} \delta(\mathbf{r})$; the 


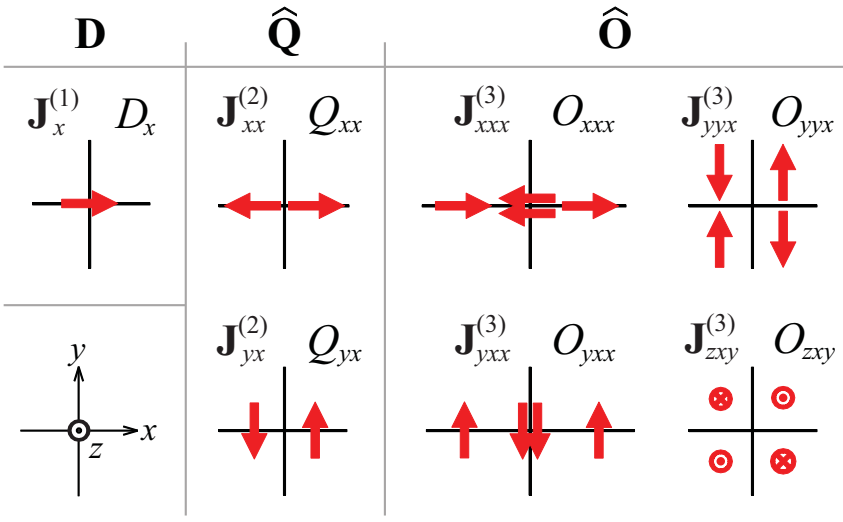

FIG. 1. Examples of elementary electric-current configurations (red arrows) in the dipole (D), quadrupole $(\hat{\mathbf{Q}})$, and octupole $(\hat{\mathbf{O}})$ excitations. Each configuration is labeled with the corresponding current component (left) and multipole tensor element (right) defined with respect to the coordinate system shown in the lower left corner.

amplitude of the second-order (quadrupole) point-current density is $\mathbf{J}^{(2)}(\mathbf{r})=i \omega \hat{\mathbf{Q}} \cdot \nabla \delta(\mathbf{r})$, where $\hat{\mathbf{Q}}$ is a current quadrupole dyadic; the octupole current density is given by $\mathbf{J}^{(3)}(\mathbf{r})=$ $-i \omega(\hat{\mathbf{O}} \cdot \nabla) \cdot \nabla \delta(\mathbf{r})$, where $\hat{\mathbf{O}}$ is an octupole triadic; and so on [16-18]. The multipoles are not divided into electric and magnetic multipoles, but can be related to them if needed. The electric current configurations of a few lowest-order multipoles are illustrated in Fig. 1. The multipole tensors $\hat{\mathbf{M}}^{(l)}$ containing the amplitudes of the $l$ th-order multipole moments are given by

$$
\mathbf{M}^{(l)}=\frac{i}{(l-1) ! \omega} \int_{V} \mathbf{J}(\mathbf{r}) \mathbf{r}^{l-1} d^{3} r .
$$

The integral in the above expression contains the outer product of $l$ vectors, one vector $\mathbf{J}(\mathbf{r})$ that is the scattering current density [16] and $l-1$ coordinate vectors $\mathbf{r}$. The integration is taken over the volume of the particle. The multipole tensors are written in Cartesian coordinates. The indices of the tensor elements show the directions of the elementary currents composing the multipole moments and their displacements with respect to each other. For example, the octupole tensor element $O_{y x x}$ stands for a $y$-directed current element (first subindex) split and symmetrically displaced along the $x$ direction two times (second and third subindices), each time flipping the negative- $x$ copy of the current elements (see Fig. 1). The distance between the current elements in each point multipole is assumed to approach zero.

In the expansion, all possible electromagnetic excitations are represented by electric currents and any dynamic magnetization effects are replaced by current loop excitations or neglected, as can be done at optical and higher frequencies [20]. This makes the classical magnetic dipole (a current loop) and electric quadrupole belong to excitations of the same order. The two classical multipoles are given by combinations of electric-current quadrupole elements. For example, a magnetic dipole moment pointing in the $z$ direction is composed of two current quadrupoles in such a way that its current configuration is $\mathbf{J}_{y x}^{(2)}-\mathbf{J}_{x y}^{(2)}$. A classical electric quadrupole with orthogonal currents oscillating in the $x y$ plane is given by $\mathbf{J}_{y x}^{(2)}+\mathbf{J}_{x y}^{(2)}$ [17]. The electric-current multipoles provide a (a)

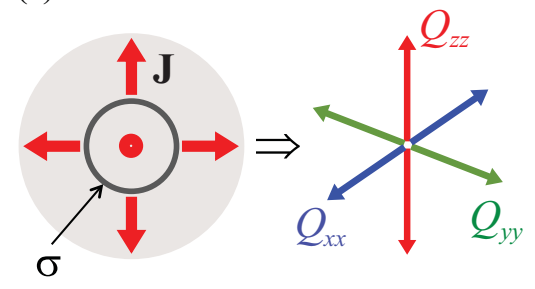

(b)

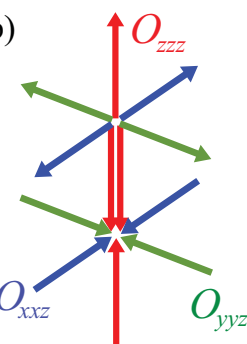

FIG. 2. Examples of anapole excitations. The fundamental anapole shown in (a) represents a radial harmonic oscillation of a spherical electron shell ( $\sigma$ is the charge density shown schematically by a dark circle and $\mathbf{J}$ is the current density shown by thick red arrows in the left picture). This excitation has three nonzero (quadrupole) moments with $Q_{x x}=Q_{y y}=Q_{z z}$. Another anapole composed of three octupoles is shown in (b). The octupole currents are equal, $J_{x x z}^{(3)}=J_{y y z}^{(3)}=J_{z z z}^{(3)}$, for which the octupole moments must satisfy $O_{x x z}=O_{y y z}=O_{z z z} / 2$.

complete orthogonal basis for expansion of localized electric currents, including also toroidal multipoles. The complexity of the electric-current configuration in the expansion increases with the multipole order. A toroidal dipole, for example, is a particular case of an electric-current octupole excitation, as will be shown later. The classical electric and magnetic multipole coefficients can be obtained from the electric-current multipole moments using a set of equations given in [16] for the coefficients up to the electric octupole.

Let us proceed to construct electromagnetic anapoles. The most symmetric (fundamental) anapole is a harmonic radial oscillation of a spherical shell of electric charge. In the current multipole expansion, this excitation is of a pure quadrupole nature, being represented by three orthogonal equal-phase and equal-amplitude linear quadrupoles $Q_{x x}, Q_{y y}$, and $Q_{z z}$ [see Fig. 2(a)]. It is remarkable to note that none of these three components is dark. Neither do they produce coinciding farfield profiles. Still the sum of the three fields is equal to zero at every point in the far-field zone. The anapole, therefore, differs considerably from the one composed of a toroidal and an ordinary dipole that have identical radiation patterns. Note also that the three quadrupole current configurations are orthogonal (being members of a complete orthogonal basis), but the fields radiated by them are not orthogonal, leading to the disappearance of the total radiated field.

It is straightforward to construct the next-order anapole by combining three octupole excitations, as shown in Fig. 2(b). The octupole moments in the illustrated example are $O_{x x z}, O_{y y z}$, and $O_{z z z}$ with equal current densities, i.e., $J_{x x z}^{(3)}=$ $J_{y y z}^{(3)}=J_{z z z}^{(3)}$. For the currents to be equal, the moments must satisfy $O_{x x z}=O_{y y z}=O_{z z z} / 2$ [see Eq. (28) in [16]]. Other multipole moments in this excitation are equal to zero. The excitation is dark, as can be seen from the fact that it is a sum of two fundamental anapoles shifted symmetrically along the $z$ axis and oscillating out of phase. There are two more anapoles of this type, one with $O_{x x x} / 2=O_{y y x}=O_{z z x}$ and the other with $O_{x x y}=O_{y y y} / 2=O_{z z y}$, which are just rotations of the anapole described above. In a similar way one can construct all the higher-order anapoles, but the complexity of the 
(a)

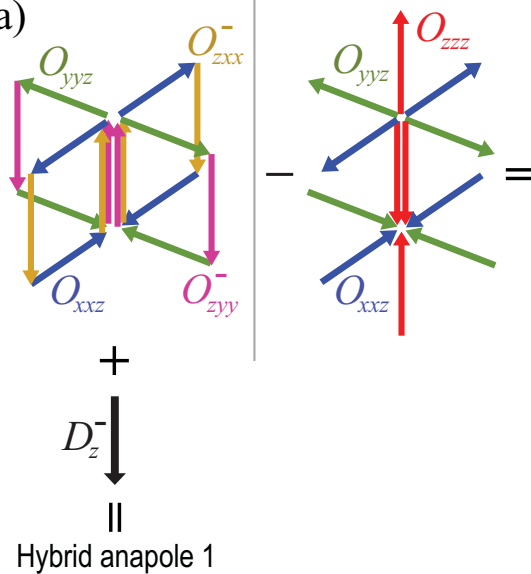

(b)

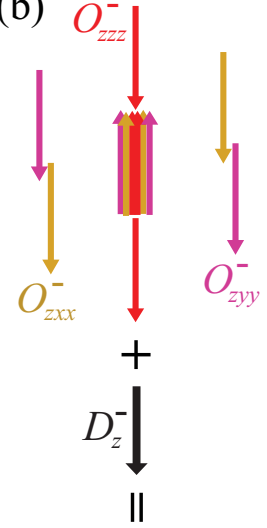

Hybrid anapole 2

FIG. 3. Construction of hybrid anapoles. Case (a) shows a combination of four equal-current octupoles forming a toroidal electric dipole. The superscript "-" indicates that the multipole moment is out of phase with the other multipole moments. Adding an ordinary field-compensating electric dipole to it results in the disappearance of the total radiation field. By subtracting a three-octupole anapole (shown in the middle) from the structure of (a), one obtains a combination of three octupoles [case (b)] that also radiates a dipolelike field. Hence, by adding an electric dipole to it, one can obtain another hybrid anapole. The octupole and dipole moments in the two hybrid anapoles obey the relations $O_{x x z}=O_{y y z}=-O_{z x x} / 2=-O_{z y y} / 2=$ $-O_{z z z} / 2$ and $D_{z}=-2 O_{x x z} k^{2}$, respectively.

current configurations in them will increase together with the difficulty to realize them in practice.

The anapoles in the above examples contain multipole moments of the same order, which makes the condition for darkness of the excitation frequency independent. In addition to these anapoles, however, one can construct hybrid anapoles that consist of different-order multipoles. The well-known toroidal-dipole-based anapole is an example of this type of an anapole (see the hybrid anapole 1 in Fig. 3). It consists of four current octupoles [forming a toroidal dipole, as seen in Fig. 3(a)] and one electric dipole. For this multipole combination to be dark, the following relation for the amplitudes of the multipole moments must be satisfied: $2 O_{x x z}=2 O_{y y z}=$ $-O_{z x x}=-O_{z y y}=-D_{z} / k^{2}$, where $k=\omega / c$ is the wave number. Obviously, this condition depends on the frequency of the excitation.

We can add or subtract any anapole excitation to or from any multipole current configuration without changing its radiated field. For example, subtracting a three-current-octupole anapole shown in the middle of Fig. 3 from the multipole combination shown in Fig. 3(a) results in a structure shown in Fig. 3(b) that radiates the same dipole field as the toroidal dipole. The obtained structure, however, consists of three current octupoles and is simpler and more symmetric than the toroidal dipole. If the excited particle is an atom, this three-octupole configuration corresponds to an out-of-phase oscillation of the charge in a spherically symmetric electron shell and in the nucleus; since the electron shell and the nucleus have opposite charges, a translational mechanical oscillation of a neutral atom as a whole must produce electromagnetic dipolelike radiation. We emphasize that the
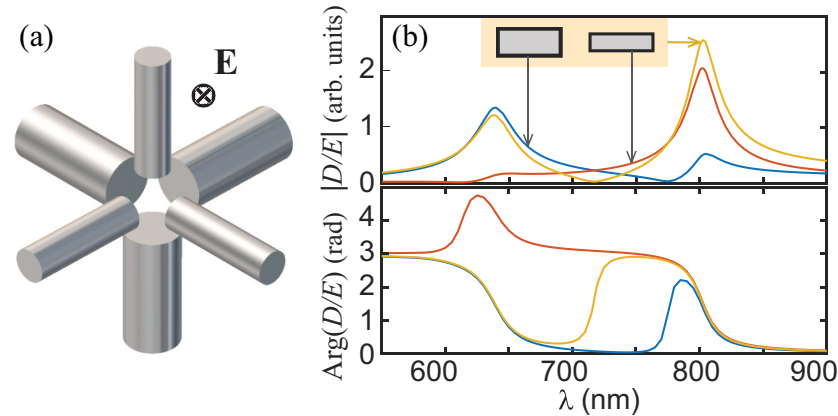

FIG. 4. Design of a silver scatterer exhibiting a fundamental anapole excitation. The structure composed of three nanorod pairs is shown in (a). The curves in (b) are the longitudinal excitation spectra of a single pair of the nanorods with equal lengths of $50 \mathrm{~nm}$, diameters of 25 and $15 \mathrm{~nm}$, and the gap of $30 \mathrm{~nm}$. The nanorods are embedded in glass. The magnitude and phase of the dipole polarizabilities of the thick nanorod (blue lines), thin nanorod (red lines), and the two nanorods (yellow line) are shown in the upper and lower plots, respectively.

volume-integrated dipole moment of this excitation is equal to zero, while its field is exactly the same as that of a dipole. Hence, this new excitation is similar in properties to a toroidal dipole. Other hybrid anapoles can also be constructed using combinations of multipoles and the considered pure or hybrid anapoles.

To realize a fundamental anapole oscillation in practice we need to excite three orthogonal linear quadrupoles shown in Fig. 2(a). If the anapole is designed to be excited optically and show a significant near-field enhancement, the particle should be chosen in the form of a plasmonic scatterer. In our design, the scatterer consists of three orthogonal pairs of cylindrical silver nanorods [see Fig. 4(a)]. The nanorods in each pair should have different geometrical parameters, such as diameters, making it possible to obtain opposite currents in them at a frequency between the two plasmon-resonance frequencies of the rods. To excite each pair equally, the excitation field must then have equal projections onto each pair. Therefore, the scatterer must be excited with a linearly polarized field that oscillates along the threefold rotational symmetry axis of the structure, as the field $\mathbf{E}$ shown in Fig. 4(a).

We first optimize the geometry of a single nanorod pair. Figure 4(b) shows the longitudinal excitation spectra of an optimized nanorod-pair structure, for which the nanorods have equal lengths of $50 \mathrm{~nm}$, but different diameters of 25 and $15 \mathrm{~nm}$. The gap between the rods is $30 \mathrm{~nm}$. The curves have been calculated using the COMSOL MULTIPHYSICS software. The optical parameters of silver were taken from [21]. The spectra show the normalized absolute value (upper curves) and phase (lower curves) of the dipole polarizability of the thick nanorod (blue lines), thin nanorod (red lines), and the combination of the two nanorods (yellow lines). The individual-nanorod curves were calculated for the two-nanorod structure, so that the interaction between the nanorods is taken into account. It can be seen that the polarizabilities of the nanorods in the pair are equal in magnitude and phase-shifted from each other approximately by $\pi$ at a wavelength of about $717 \mathrm{~nm}$. At this wavelength, the dipole 
(a)

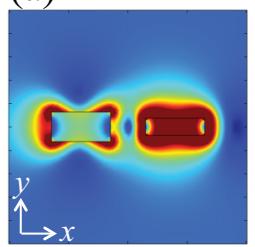

(d)

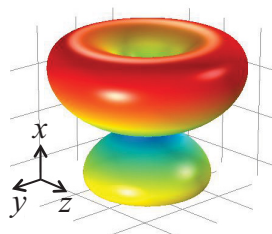

(g)

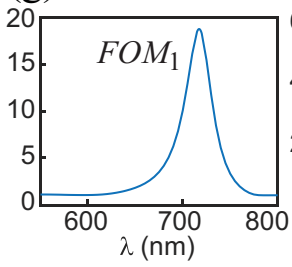

(b)

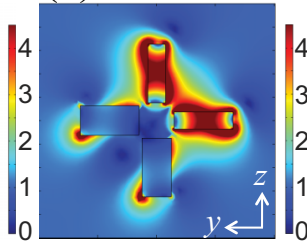

(e)

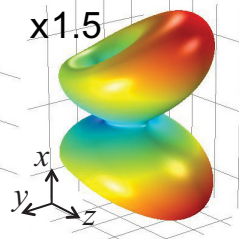

(h)

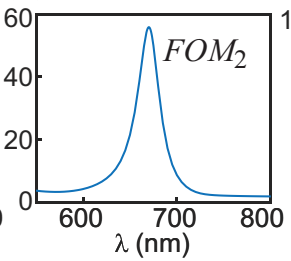

(c)

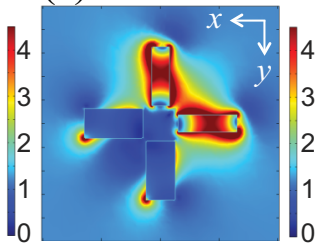

(f)

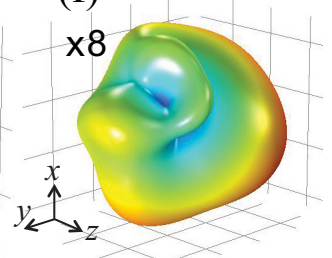

(i)

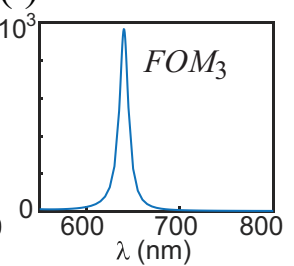

FIG. 5. Radiation characteristics of the designed scatterers: (a), (b), and (c) show the electric-field amplitude distributions in the near-field zone of a one-, two-, and three-nanorod-pair structure, respectively (the area shown is $100 \times 100 \mathrm{~nm}^{2}$ and the amplitude is normalized to that of the incident field); (d), (e), and (f) illustrate the corresponding far-field amplitude distributions magnified in (e) by a factor of 1.5 and in (f) by a factor of 8 ; (g), (h), and (i) illustrate the spectra of the figure of merit $\mathrm{FOM}_{i}$ given by Eq. (2) for the three structures, where $i$ is the number of nanorod pairs in the structure.

polarizability of the pair vanishes, manifesting the electricdipole suppression of the structure [17,18,22]. The curves were calculated in the same way as in [17].

The scattering properties of two and three orthogonal nanorod pairs were studied using COMSOL MULTIPHYSICS as well. We have found that the scattering suppression effect is present in these structures at slightly different wavelengths, which is explained by the nanorod interaction. For the twopair structure, the excitation field was chosen to propagate normally to the plane of the four rods and to be polarized symmetrically with respect to the two nanorod pairs, i.e., at $45^{\circ}$ to the nanorod axes. The scattering was suppressed at $\lambda=670 \mathrm{~nm}$. For the six-nanorod structure, we used a two-beam excitation, with one beam exciting two nanorod pairs exactly as in the above example and the second beam propagating perpendicularly to the first beam and polarized longitudinally with respect to the third nanorod pair. The fields were made to oscillate in phase at the center of the structure and their amplitudes were selected for the total field to be polarized symmetrically with respect to the three pairs of the nanorods. The scattering in this case was suppressed at $\lambda=640 \mathrm{~nm}$. Figure 5 shows the calculation results for the three structure types. In (a), (b), and (c) we show the electric-field amplitude distribution in the structures at the radiation-suppression wavelengths. The near-field enhance- ment reaches approximately the same level in the three cases. The $x y, x z$, and $y z$ cross sections of the field distribution in the six-rod structure are very similar to each other and therefore we show only one of them here. The far-field amplitude profiles in Figs. 5(d)-5(f), illustrated respectively for the three structures, differ considerably. These profiles were calculated from the fields at $300 \mathrm{~nm}$ distance from the center of the structure by using the Stratton-Chu formula [23]. Already the single pair provides a significant suppression of scattering. The far-field profile [Fig. 5(d)] shows a slightly asymmetric radiation pattern of a vertically aligned current quadrupole $Q_{x x}$. Figure 5(e) shows the radiation pattern of the structure illustrated in Fig. 5(b). The profile is magnified (zoomed in) by a factor of 1.5 compared to the profile of Fig. 5(d). It also exhibits a small asymmetry caused by the near-field interaction of the nanorod pairs. Finally, the radiation pattern of the six-rod structure is shown in Fig. 5(f) (with the $x, y$, and $z$ axes pointing from a thinner to a thicker nanorod in each pair). It is magnified by a factor of 8 compared to the one in Fig. 5(d), because of further significant suppression of scattering.

To quantify the scattering suppression effect, we introduce the following figure of merit:

$$
\mathrm{FOM}=C_{\mathrm{D}}^{\mathrm{eqv}} / C_{\mathrm{tot}},
$$

where $C_{\mathrm{tot}}$ is the total scattering cross section of the designed particle (proportional to the total optical power radiated by the electric current density excited in the particle) and $C_{\mathbf{D}}^{\mathrm{eqv}}$ is the equivalent scattering cross section of a point dipole with the same integrated magnitude of the electric current density. In other words, FOM is a scattering suppression factor relative to a pure dipole excitation. Figures 5(g)-5(i) show the spectra of FOM calculated for one, two, and three nanorod pairs, respectively. The scattering suppression factor reaches the value of 19 for a single nanorod pair, 56 for the two pairs, and 970 for the three nanorod pairs designed to realize the anapole. The obtained suppression factor is rather high, considering that we did not reoptimize the geometry of each nanorod pair in order to compensate for the effect of near-field interaction between them. This implies that the scattering by the threepair anapole structure will be very dark even if some design and/or fabrication errors will be present in its experimental realization.

In summary, we applied multipole expansion of localized electric currents in Cartesian coordinates to construct electromagnetic anapoles. Some of the anapoles are pure in a sense that they include only current multipole moments of the same order. The simplest of them is a centrosymmetric anapole that can be decomposed into three orthogonal current quadrupoles with tensor elements $Q_{x x}=Q_{y y}=Q_{z z}$. This fundamental anapole can be seen as a building block for other pure anapoles. We have proposed a plasmonic realization of this anapole and studied its properties. We have found that, using an anapole excitation in a six-nanorod scatterer, the scattering can easily be suppressed by three orders of magnitude. We have also demonstrated the construction of hybrid anapoles that include current multipoles of different orders. One of such anapoles includes four octupoles and one dipole. It coincides with a well-known anapole based on a toroidal dipole. We have also obtained another, simpler and 
more symmetric hybrid anapole composed of three octupoles and one dipole. The three-octupole structure has the same scattering properties as the toroidal-dipole configuration. The new excitation represents an out-of-phase oscillation of charge in a fixed-radius spherical shell and an equal charge at the shell center. The considered anapole oscillations can be realized and tested in practice, especially at low frequencies, and possibly also found in nature. We believe that the study and use of the introduced anapoles can in the future bring about new possibilities for electromagnetic sensing, detection, and communication.

This research was funded by the Academy of Finland (Project No. 308394). We also acknowledge the Academy of Finland Flagship Programme, Photonics Research and Innovation (PREIN, 320167). V.V. additionally acknowledges Narodowe Centrum Nauki, Poland (PRELUDIUM-16, Grant No. 2018/31/N/ST7/03918).
[1] S. Nanz, Toroidal Multipole Moments in Classical Electrodynamics (Springer Spektrum, Wiesbaden, 2016).

[2] V. M. Dubovik and V. V. Tugushev, Toroid moments in electrodynamics and solid-state physics, Phys. Rep. 187, 145 (1990).

[3] G. N. Afanasiev and V. M. Dubovik, Electromagnetic properties of a toroidal solenoid, J. Phys. A 25, 4869 (1992).

[4] T. Kaelberer, V. A. Fedotov, N. Papasimakis, D. P. Tsai, and N. I. Zheludev, Toroidal dipolar response in a metamaterial, Science 330, 1510 (2010).

[5] K. Sawada and N. Nagaosa, Optical Magnetoelectric Effect in Multiferroic Materials: Evidence for a Lorentz Force Acting on a Ray of Light, Phys. Rev. Lett. 95, 237402 (2005).

[6] K. Marinov, A. D. Boardman, V. A. Fedotov, and N. Zheludev, Toroidal metamaterial, New J. Phys. 9, 324 (2007).

[7] G. N. Afanasiev and V. M. Dubovik, Some remarkable chargecurrent configuration, Phys. Part. Nucl. 29, 366 (1998).

[8] A. D. Boardman, K. Marinov, N. Zheludev, and V. A. Fedotov, Dispersion properties of nonradiating configurations: Finitedifference time-domain modeling, Phys. Rev. E 72, 036603 (2005).

[9] N. A. Nemkov, A. A. Basharin, and V. A. Fedotov, Nonradiating sources, dynamic anapole, and Aharonov-Bohm effect, Phys. Rev. B 95, 165134 (2017).

[10] N. Papasimakis, V. A. Fedotov, V. Savinov, T. A. Raybould, and N. I. Zheludev, Electromagnetic toroidal excitations in matter and free space, Nat. Mater. 15, 263 (2016).

[11] L. Xu, M. Rahmani, K. Z. Kamali, A. Lamprianidis, L. Ghirardini, J. Sautter, R. Camacho-Morales, H. Chen, M. Parry, I. Staude, G. Zhang, D. Neshev, and A. E. Miroshnichenko, Boosting third-harmonic generation by a mirror-enhanced anapole resonator, Light Sci. Appl. 7, 44 (2018).
[12] K. V. Baryshnikova, D. A. Smirnova, B. S. Luk'yanchuk, and Y. S. Kivshar, Optical anapoles: Concepts and applications, Adv. Opt. Mater. 7, 1801350 (2019).

[13] A. E. Miroshnichenko, A. B. Evlyukhin, Y. F. Yu, R. M. Bakker, A. Chipouline, A. I. Kuznetsov, B. Luk'yanchuk, B. N. Chichkov, and Y. S. Kivshar, Nonradiating anapole modes in dielectric nanoparticles, Nat. Commun. 6, 8069 (2015).

[14] Y. Yang, V. A. Zenin, and S. I. Bozhevolnyi, Anapole-assisted strong field enhancement in individual all-dielectric nanostructures, ACS Photonics 5, 1960 (2018).

[15] G. Grinblat, Y. Li, M. P. Nielsen, R. F. Oulton, and S. A. Maier, Enhanced third harmonic generation in single germanium nanodisks excited at the anapole mode, ACS Nano 11, 953 (2017).

[16] P. Grahn, A. Shevchenko, and M. Kaivola, Electromagnetic multipole theory for optical nanomaterials, New J. Phys. 14, 093033 (2012).

[17] P. Grahn, A. Shevchenko, and M. Kaivola, Electric dipole-free interaction of visible light with pairs of subwavelength-size silver particles, Phys. Rev. B 86, 035419 (2012).

[18] A. Shevchenko, V. Kivijärvi, P. Grahn, M. Kaivola, and K. Lindfors, Bifacial Metasurface with Quadrupole Optical Response, Phys. Rev. Appl. 4, 024019 (2015).

[19] E. Hurwitz and G. Gbur, Null-field radiationless sources, Opt. Lett. 39, 6529 (2014).

[20] L. D. Landau and E. M. Lifshitz, Electrodynamics of Contiuous Media (Pergamon, Oxford, 1984).

[21] P. B. Johnson and R. W. Christy, Optical constants of the noble metals, Phys. Rev. B 6, 4370 (1972).

[22] P. Grahn, A. Shevchenko, and M. Kaivola, Multipole polarizability of a nanodimer in optical waves, J. Eur. Opt. Soc. Rapid Publ. 8, 13009 (2013).

[23] J. A. Stratton and L. J. Chu, Diffraction theory of electromagnetic waves, Phys. Rev. 56, 99 (1939). 